\title{
GALERKIN'S METHOD FOR BOUNDARY INTEGRAL EQUATIONS ON POLYGONAL DOMAINS
}

\author{
G. A. CHANDLER'
}

(Received 19 January 1983; Revised 23 May 1983)

\begin{abstract}
A harmonic function in the interior of a polygon is the double layer potential of a distribution satisfying a second kind integral equation. This may be solved numerically by Galerkin's method using piecewise polynomials as basis functions. But the corners produce singularities in the distribution and the kernel of the integral equation; and these reduce the order of convergence. This is offset by grading the mesh, and the orders of convergence and superconvergence are restored to those for a smooth boundary.
\end{abstract}

\section{Introduction}

Boundary integral methods are used extensively in the numerical solution of potential problems in two and three dimensions. (See Christiansen and Hansen [11], Jaswon and Symm [16], Brebbia [7] for example.) Here we consider the simplest case:- Dirichlet's problem for Laplace's equation in a two dimensional domain. The solution can be obtained from the double layer distribution, which in turn can be found as the solution of a second kind integral equation. If the domain has a smooth boundary, the integral operator will be compact and the distribution will be smooth. The standard theory (Atkinson [2], Baker [6]) then establishes the order of convergence of the chosen numerical method. When the domain has corners the kernel of the integral operator contains singularities which prevent it being compact, and even for smooth boundary data the double layer distribution will contain singularities. Both problems need to be considered if a numerical method is to retain its order of convergence.

\footnotetext{
' Department of Mathematics, University of Queensland, St. Lucia, Queensland 4067.

(C) Copyright Australian Mathematical Society 1984, Serial-fee code 0334-2700/84
} 
A frequent remedy is to add the singularities appearing in the distribution to the underlying set of basis functions (Jaswon and Symm [16], Djaoria [13]). Here we investigate the alternative of grading the mesh. This approach, which is used extensively in finite element computations and in solving ordinary differential equations, is simpler to implement. It has been investigated for weakly singular integral equations by Chandler [9], Graham [14] and Schneider [22].

We suppose that the boundary integral equations are solved by Galerkin's method using piecewise polynomials of degree $r$ as basis functions. In the case of a smooth boundary this method produces an order $r+1$ approximation to the double layer distribution (i.e. if $n$ is the number of mesh points used, the error is $\left.O\left(1 / n^{r+1}\right)\right)$. Superconvergence phenomena can then be used to simply compute an order $2 r+2$ approximation (Chandler [8], Lin Qun [20]). With corners the rate of convergence is limited by the angle of the corner (it will always be less than $3 / 2$ ). By grading the mesh sufficiently in the neighbourhood of the corners the order of convergence may be restored to $r+1$. Moreover an even more pronounced grading restores the full superconvergence of the Galerkin solution. This gives an order $2 r+2$ approximation to the double layer potential, and hence to the original harmonic problem. (Thus an order 4 approximation is obtained from piecewise linear approximation.)

In Section 2 the boundary integral equation is formulated to display the nature of the non-compact parts. In Section 3, we prove results about these non-compact operators and their numerical approximation. A by-product of this analysis is a simple proof of the regularity results needed for the double layer potential. Section 4 applies the results of Section 3 to prove orders of convergence for the boundary integral equation.

\section{Formulation}

Consider the interior Dirichlet problem

$$
\begin{gathered}
\frac{\partial^{2} U}{\partial x_{1}^{2}}+\frac{\partial^{2} U}{\partial x_{2}^{2}}=0, \quad\left(x_{1}, x_{2}\right) \in \Omega, \\
\left.U\right|_{\Gamma}=g,
\end{gathered}
$$

where $\Omega \subset \mathbf{R}^{2}$ is the interior of the region bounded by a simple closed polygon $\Gamma$. Let

$$
G(x, \xi)=\frac{1}{2 \pi} \ln |x-\xi|, \quad x, \xi \in \bar{\Omega},
$$

be the fundamental solution to Laplace's equation, and let $G(x, \xi)^{\prime}, x \in \bar{\Omega}, \xi \in \Gamma$ denote the normal derivative of $G$ with respect to $\xi$ (the positive normal is 
assumed to point outwards). It is known (Kellogg [17], Král [19], Cryer [12]) that

$$
U(x)=\left(\Upsilon_{u}\right)(x)=\int_{\Gamma} G(x, \xi)^{\prime} u(\xi) d \xi, \quad x \in \bar{\Omega},
$$

where $u$, the double layer distribution, satisfies the second kind integral equation

$$
u(x)+2(\mathcal{V} u)(x)=f(x), \quad x \in \Gamma,
$$

with $f=2 g$. For convenience, suppose $\Gamma$ is parametrized by arc length $s$, with corner points at $0=s_{1}, s_{2}, \ldots, s_{m}$. No notational distinction is made between $s$ and the point in $\mathbf{R}^{2}$ of length $s$ around $\Gamma$, and we write $u(s)$ or

$$
\int_{\Gamma} G(s, \sigma)^{\prime} u(\sigma) d \sigma
$$

(for the right-hand side of (2.1)) for example. For each $i, \Gamma_{i}$ denotes the side of $\Gamma$ with $s_{i-1}<s<s_{i}$. (Throughout the obvious periodicity convention is adopted, with $s_{m+1}$ and $\Gamma_{m+1}$ denoting $s_{1}$ and $\Gamma_{1}$ respectively.) At each $s_{i}$, the number $\chi_{i} \in(-1,1)$ is defined by requiring $\left(1-\chi_{i}\right) \pi$ to be the angle $s_{i-1} \hat{s}_{i} s_{i+1}$.

As $u$ may be discontinuous at the corners, it is convenient to regard a double layer distribution as a vector of functions $\left(u_{1}, \ldots, u_{m}\right), u_{i}: \bar{\Gamma}_{i} \rightarrow \mathbf{R}$. Then (2.2) may be rewritten as an $m \times m$ system of integral equations

$$
(q+F) \mathbf{u}=\mathbf{f}
$$

where $\mathbf{u}=\left(u_{1}, \ldots, u_{m}\right), \mathbf{f}=\left(f_{1}, \ldots, f_{m}\right)$ with $f_{i}$ a continuous extension of $\left.f\right|_{\Gamma_{i}}$ to $\bar{\Gamma}_{i}$; and where $\mathcal{G}$ is defined by

$$
(\mathcal{G u})_{i}(s)=2 \sum_{j} \int_{\Gamma_{j}} G(s, \boldsymbol{\sigma})^{\prime} u_{j}(\boldsymbol{\sigma}) d \boldsymbol{\sigma}, \quad s \in \Gamma_{i} .
$$

If then a double layer distribution $u: \Gamma \rightarrow \mathbf{R}$ satisfies $\left.u\right|_{\Gamma_{i}}=\left.u_{i}\right|_{\Gamma_{i}}$, its double layer potential solves the original Dirichlet problem. (The values $u\left(s_{1}\right), \ldots, u\left(s_{m}\right)$ are not important). Given this identification is understood, we write $u$ for $\mathbf{u}$ subsequently.

The kernel of $\mathcal{G}$ can now be explicitly calculated. Suppose $s \in \Gamma_{i}$. Then if $\sigma \in \Gamma_{i}, G\left(s_{i}, \sigma\right)^{\prime}=0$. If $\sigma \notin \Gamma_{i-1} \cup \Gamma_{i+1} G(s, \sigma)^{\prime}$ is a smooth function of $s$ and $\sigma$. Otherwise

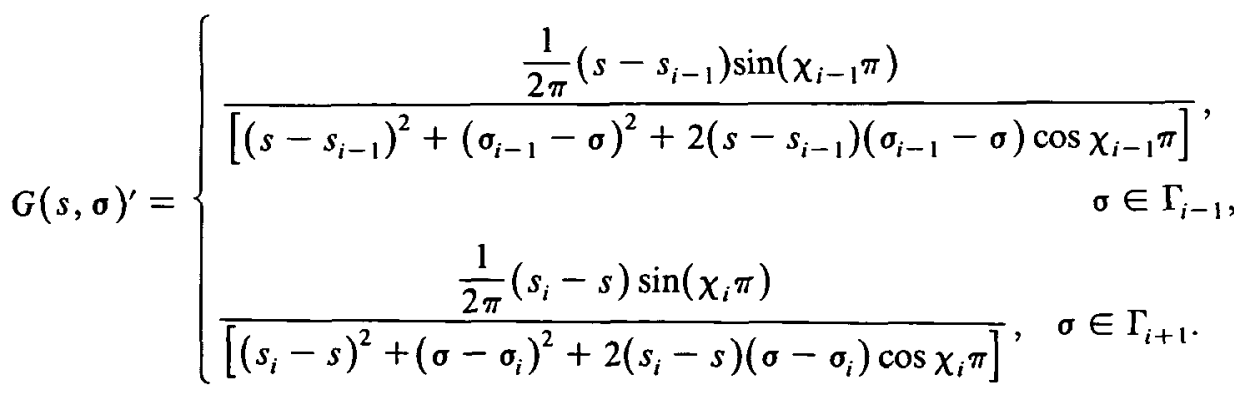


Thus $\mathcal{G}=\Re+\mathscr{K}$ where for $s \in \Gamma_{i}$

$$
(\Re u)_{i}(s)=2 \int_{\Gamma_{i-1}} G(s, \sigma)^{\prime} u(\sigma) d \sigma+2 \int_{\Gamma_{i+1}} G(s, \sigma)^{\prime} u(\sigma) d \sigma
$$

and where $\mathscr{K}$ has a smooth kernel. This decomposition is a particular case of the more general results in Cryer [12] or Král [19]. Finally observe that $(\mathscr{R} u)_{i}(s)$ has not been defined for $s \in\left\{s_{i-1}, s_{i}\right\}$. The conventions

$$
\begin{gathered}
(\Re u)_{i}\left(s_{i}\right)=u_{i+1}\left(s_{i}\right) \chi_{i}+2 \int_{\Gamma_{i-1}} G\left(s_{i}, \sigma\right)^{\prime} u(\sigma) d \sigma, \\
(\Re u)_{i}\left(s_{i-1}\right)=u_{i-1}\left(s_{i-1}\right) \chi_{i-1}+2 \int_{\Gamma_{i+1}} G\left(s_{i-1}, \sigma\right)^{\prime} u(\sigma) d \sigma,
\end{gathered}
$$

will ensure that each $(\Re u)_{i}$ is continuous if each $u_{i}$ is continuous.

\section{Preliminary results}

For real numbers $\chi \in(-1,1)$ and $p$ let

$$
R_{p, \chi}(s, \sigma)=\frac{\sin (\chi \pi) s^{1-p_{\sigma} p}}{\pi\left(s^{2}+\sigma^{2}+2 s \sigma \cos \chi \pi\right)} .
$$

Define an integral operator by

$$
\left(\Re_{p, \chi} u\right)(s)=\left\{\begin{array}{l}
\int_{0}^{1} R_{p, \chi}(s, \sigma) u(\sigma) d \sigma, \quad 0<s \leqslant 1, \\
u(0) \frac{\sin \chi \pi p}{\sin \pi p}, \quad s=0, p \neq 0, \\
u(0) \chi, \quad s=p=0 .
\end{array}\right.
$$

If $u$ is continuous, then so is $\Re_{p, x} u$. The non-compact parts of $\mathcal{G}$ are of the form (3.1) with $p=0$. The extension of results from $\Re_{p, x}$ to the matrix of operators $R$ is straightforward, except for notation. Thus we give the essential results about $\Re_{p, x}$ here, with a more concise suggestion of their extension in Section 4.

Equation (3.1) defines an integral operator on a variety of function spaces. Most importantly we need $L^{2}(I)$, the Hilbert space of square integrable functions on $I=[0,1]$ with the usual norm denoted by $\|\cdot\|$. We also need the Banach space $\mathcal{C}(I)$ of continuous functions on $I$ with the supremum norm $\|\cdot\|_{\infty}$. Appropriate bounds for $\left\|\Re_{p, \chi}\right\|$ are provided by the following lemmas.

LEMMA 1. For $\chi \in(-1,1)$ and $p=0, \Re_{0, \chi}$ is a bounded operator on $L^{2}(I)$, with $\left\|\Re_{0, \chi}\right\| \leqslant|\sin (\chi \pi / 2)|$. 
Proof. An isometry $g: L^{2}(I) \rightarrow L^{2}\left(\mathbf{R}_{+}\right), \mathbf{R}_{+}=\{x \in \mathbf{R}: x \geqslant 0\}$ is defined by $(\mathscr{g} u)(t)=u\left(e^{-t}\right) e^{-t / 2}$. A change of variables gives

$$
\left(g \Re_{0, x} g^{-1} v\right)(t)=\int_{0}^{\infty} r_{\chi}(t-\tau) v(\tau) d \tau
$$

with

$$
r_{\chi}(\tau)=e^{\tau / 2} /\left(e^{2 \tau}+e^{\tau} \cos \chi \pi+1\right) .
$$

The Fourier transform of $r_{\chi}$ is

$$
\hat{r}_{\chi}(y)=\frac{\sinh \pi \chi(y-i / 2)}{\sinh \pi(y-i / 2)}
$$

The lemma follows because

$$
\left\|\Re_{0, \chi}\right\|=\left\|g \Re_{0, x} g^{-1}\right\| \leqslant \sup _{y \in \mathbf{R}}\left|\hat{r}_{\chi}(y)\right| .
$$

LEMMA 2. For $\chi \in(-1,1)$ and $p \in(-1,1), \Re_{p, \chi}$ is a bounded operator on $\mathcal{C}(I)$ and

$$
\left\|\Re_{p, \chi}\right\|= \begin{cases}|\sin (\chi \pi p) / \sin (\pi p)|, & p \neq 0 \\ |\chi|, & p=0\end{cases}
$$

PROOF. Since $\mathcal{R}_{p, \chi}$ is now operating on $\mathcal{C}(I)$,

$$
\begin{aligned}
\left\|\Re_{p, \chi}\right\| & =\sup _{s \in[0,1]}\left\{\frac{|\sin \chi \pi|}{\pi} \int_{0}^{1} \frac{s^{1-p_{\sigma}}}{s^{2}+\sigma^{2}+2 s \sigma \cos \chi \pi} d \sigma\right\} \\
& =\frac{|\sin \chi \pi|}{\pi} \int_{0}^{\infty} \frac{\sigma^{p}}{\sigma^{2}+1+2 \sigma \cos \chi \pi} d \sigma
\end{aligned}
$$

gives the required equality.

As the operator $\Re$ of Section 2 must be applied to functions with singularities at the corners, we need an appropriate space to describe them. Thus define $e_{\alpha}^{k}\left(I^{-}\right), \alpha>0$, as the Banach space of continuous functions on $I$ whose $k$ th derivatives exist on $(0,1]$, and for which the norm

$$
\|u\|_{k, \alpha}= \begin{cases}\max \left\{\|u\|_{\infty}, \sup \left\{\left|s^{k-\alpha}\left(D^{k} u\right)(s)\right|: 0<s \leqslant 1\right\}\right\}, & k>\alpha \\ \max \left\{\|u\|_{\infty},\left\|D^{k} u\right\|_{\infty}\right\}, & k \leqslant \alpha\end{cases}
$$

is finite. The space $e_{\alpha}^{k}\left(I^{+}\right)$is defined analogously, but with $(1-s)^{k-\alpha}$. Observe that the condition $\|u\|_{k, \alpha}<\infty$ excludes singularities of the form $s^{\beta}$, when $\beta<\alpha$, but allows them for $\beta>\alpha$. 
The results of Lemma 2 can be used to establish the smoothness of the solution to the equation

$$
\left(\mathscr{G}+\Re_{0, x}\right) v_{0}=f .
$$

This is most easily expressed by defining $B$ to be the Banach space $B=\mathbf{R} \times$ $\mathcal{C}((0,1])$ with norm $\|(\gamma, w)\|=\max \left\{|\gamma|,\|w\|_{\infty}\right\}$. Let $\mathcal{E}$ be the topological isomorphism

$$
\varepsilon: \mathcal{C}_{\alpha}^{1}\left(I^{-}\right) \rightarrow B: u \rightarrow\left(u(0), \tilde{D}_{\alpha} u\right)
$$

where $\left(\tilde{D}_{\alpha} u\right)(s)=s^{1-\alpha} D u(s)$. Define two operators $\delta$ and $\mathcal{K}$ on $B$ by

$$
\begin{aligned}
\mathcal{S}(\gamma, w) & =\left(\chi \gamma, \Re_{\left.\alpha, \chi^{w}\right),}\right. \\
\mathcal{K}(\gamma, w) & =\left(0, w_{\alpha} \mathscr{K}_{1}(\gamma, w)\right), \\
w_{\alpha}(s) & =\frac{\sin (\chi \pi) s^{1-\alpha}}{\pi\left(1+s \cos \chi \pi+s^{2}\right)}, \\
\left(\mathcal{K}_{1}(\gamma, w)\right)(s) & =\gamma+\int_{0}^{s} \sigma^{\alpha-1} w(\sigma) d \sigma .
\end{aligned}
$$

Whenever $v \in \mathrm{C}_{\alpha}^{\mathrm{l}}\left(I^{-}\right)$,

$$
\tilde{D}_{\alpha} \Re_{0, \chi} v=\Re_{\alpha, \chi} \tilde{D}_{\alpha} v-\mathscr{K}_{1}\left(v(0), \tilde{D}_{\alpha}(v)\right)(1) w_{\alpha},
$$

and thus

$$
(G+\delta+\mathcal{K})=\mathcal{E}\left(\mathscr{G}+\Re_{0, \chi}\right) \mathcal{E}^{-1} .
$$

Because $\mathscr{G}+\mathscr{R}_{0, \chi}$ is $1-1$, so is $9+\Im+\mathscr{K}$. If $\alpha<1 /(1+|\chi|)$ then Lemma 2 can be used to show that $\delta$ is a contraction (i.e. $\|\delta\|=\left\|\Re_{\alpha, \chi}\right\|<1$ ). Thus as $\mathscr{K}$ is compact, the Fredholm alternative shows that $\mathscr{G}+\delta+\mathscr{K}$ is invertible on $B$. Therefore if $f \in \mathcal{C}_{\alpha}^{1}\left(I^{-}\right), \mathcal{E}^{-1}(g+\delta+\mathcal{K})^{-1} \mathcal{E} f$ is the unique solution of (3.2) on $\mathcal{C}(I)$. Hence

$$
\left\|v_{0}\right\|_{1, \alpha}=\left\|\mathcal{E}^{-1}(\mathcal{G}+\mathcal{S}+\mathcal{K})^{-1} \mathcal{\delta}\right\| \leqslant C\|f\|_{1, \alpha} .
$$

This argument may be continued to higher derivatives to show that for all $k \geqslant 1$ and $\alpha<1 /(1+|\chi|)$ that

$$
\left\|v_{0}\right\|_{k, \alpha} \leqslant C\|f\|_{k, \alpha}
$$

(with $C$ independent of $f$ ). Or more succinctly, $g+\Re_{0, x}$ has a bounded inverse on $\mathcal{C}_{\alpha}^{k}\left(I^{-}\right)$.

More generally equation (3.2) may be replaced with the equation

$$
\left(9+\Re_{0, x}+\mathcal{K}_{0}\right) v_{0}=f \text {, }
$$


for any operator $\mathscr{K}_{0}$ with a smooth kernel. The above reasoning shows that if $\left(\mathcal{G}+\mathfrak{R}_{0, \chi}+\mathscr{K}_{0}\right)$ is invertible on $C(I)$, it is also invertible on $\mathcal{C}_{\alpha}^{k}\left(I^{-}\right)$provided $\alpha<1 /(1+|x|)$.

If $u \in \mathcal{C}_{\alpha}^{k}\left(I^{-}\right)$is to be approximated by piecewise polynomials, the mesh must generally be non-uniform with more break points near zero. For any $q>0$ ( $q$ will be termed the grading exponent) define the mesh $\left\{\sigma_{0}, \ldots, \sigma_{n}\right\}$ by

$$
\sigma_{i}=(i / n)^{q} .
$$

Let $S_{n}$ be the set of piecewise polynomials of degree $r$ which are possibly discontinuous at the $\sigma_{i}$. Let $P_{n}$ denote the orthogonal projection of $L^{2}(I)$ onto $S_{n}$ and $P_{n}^{\prime}=\mathscr{G}-P_{n}$. Using the notation $I_{i}=\left(\sigma_{i-1}, \sigma_{i}\right), h_{i}=\left(\sigma_{i}-\sigma_{i-1}\right)$ it can be verified that

$$
h_{i}<C i^{q-1} / n^{q}
$$

and

$$
\left\|P_{n}^{\prime} u\right\|_{i, \infty}= \begin{cases}C h_{i}^{r+1}\left\|D^{r+1} u\right\|_{i, \infty}, & i>1, \\ C h_{i}^{\alpha}\|u\|_{r+1, \alpha}, & i=1\end{cases}
$$

with the constants $C$ independent of $n, u$ and $i$; and where $\|\cdot\|_{i, \infty}$ denotes the supremum norm over the subinterval $I_{i}$. (As usual the constant $C$ may be different in different places.)

The inequalities (3.4)-(3.6) suffice to derive the well-known result that if $u \in \mathcal{C}_{\alpha}^{r+1}\left(I^{-}\right), \alpha \leqslant r+1$, and if $q>(r+1) /\left(\alpha+\frac{1}{2}\right)$, then

$$
\left\|P_{n}^{\prime} u\right\| \leqslant \frac{C}{n^{r+1}}\|u\|_{r+1, \alpha}
$$

(Rice [21] for example). Thus a sufficient grading of the mesh near zero compensates for the effect of singularities and restores the order $r+1$ convergence of $P_{n} u$ to $u$ (The rate expected when $u$ is smooth and a uniform mesh is used.) It is further known that if an integral operator $\mathscr{K}_{0}$ has a smooth kernel then $\left\|\mathcal{K}_{0} P_{n}^{\prime} u\right\|$ $=O\left(1 / n^{2 r+2}\right)$ (Chandler [8], Lin Qun [20]) when the mesh is uniform and $u$ is smooth. If $u \in \mathbb{C}_{\alpha}^{r+1}\left(I^{-}\right)$the condition $q>(r+1) /\left(\alpha+\frac{1}{2}\right)$ is again sufficient to restore $\left\|\mathscr{K}_{0} P_{n}^{\prime} u\right\|=O\left(1 / n^{2 r+2}\right)$. But for the operator $\Re_{0, x}$ an even stronger grading is required (because of the singularities in the kernel).

LEMMA 3. If $u \in \mathcal{C}_{\alpha}^{r+1}\left(I^{-}\right), \alpha \leqslant r+1$, and $q>(2 r+2) /\left(\alpha+\frac{1}{2}\right)$ then $\left\|\Re_{0, \chi} P_{n}^{\prime} u\right\| \leqslant O\left(1 / n^{2 r+2}\right)$. 
Proof. Defining the function $R_{s}$ by $R_{s}(\sigma)=R_{0, x}(s, \sigma)$,

$$
\begin{aligned}
\left|\left(\Re_{0, x} P_{n}^{\prime} u\right)(s)\right| & =\left|\left(R_{s}, P_{n}^{\prime} u\right)\right|=\left|\left(P_{n}^{\prime} R_{s}, P_{n}^{\prime} u\right)\right| \\
& \leqslant\left|\int_{I_{1}} R_{s} P_{n}^{\prime} u\right|+\sum_{i>1} \int_{I_{i}}\left|P_{n}^{\prime} R_{s}\right|\left|P_{n}^{\prime} u\right| \\
& =T_{1}+T_{2} .
\end{aligned}
$$

It is straightforward to show

$$
\left|\left(D^{r+1} R_{s}\right)(\sigma)\right| \leqslant C \min \left\{\frac{1}{s^{r+2}}, \frac{1}{\sigma^{r+2}}\right\}=\Phi(s, \sigma)
$$

where $C$ depends only on $\chi$ and $r$. Then the second term of (3.8) may be bounded using (3.4) and (3.5) to give

$$
\begin{aligned}
T_{2} & \leqslant C \sum_{i>1} h_{i}\left\|P_{n}^{\prime} R_{s}\right\|_{i, \infty}\left\|P_{n}^{\prime} u\right\|_{i, \infty} \\
& \leqslant C \sum_{i>1} h_{i}^{2 r+3}\left\|D^{r+1} R_{s}\right\|_{i, \infty}\left\|D^{r+1} u\right\|_{i, \infty} \\
& \leqslant \frac{C\|u\|_{r+1, \alpha}}{n^{2 r+2}} \sum_{i>1} \frac{1}{n}\left(\frac{i}{n}\right)^{q(\alpha+r+2)-2 r-3} \Phi\left(s,\left(\frac{i}{n}\right)^{q}\right) \\
& \leqslant \frac{C\|u\|_{r+1, \alpha}}{n^{2 r+2}} \int_{0}^{1} \sigma^{q(\alpha+r+2)-2 r-3} \Phi\left(s, \sigma^{q}\right) d \sigma \\
& \leqslant \frac{C\|u\|_{r+1, \alpha}}{n^{2 r+2}}\left(1+s^{\alpha-2(r+1) / q}\right) .
\end{aligned}
$$

To bound the first term of (3.8), use (3.6) to see

$$
T_{1} \leqslant \int_{I_{1}}\left|R_{s}\right|\left|P_{n}^{\prime} u\right| \leqslant C\left\|P_{n}^{\prime} u\right\|_{1, \infty} \leqslant C h_{1}^{\alpha}\|u\|_{1, \alpha},
$$

or alternatively when $s>h_{1}$,

$$
T_{1} \leqslant \int_{I_{1}}\left|P_{n}^{\prime} R_{s}\right|\left|P_{n}^{\prime} u\right| \leqslant \frac{C h_{1}^{\alpha+r+2}}{s^{r+2}}\|u\|_{1, \infty} .
$$

Thus

$$
T_{1} \leqslant \frac{C\|u\|_{r+1, \alpha}}{n^{q(r+2+\alpha)}} \min \left\{\frac{1}{s^{r+2}}, n^{q(r+2)}\right\} .
$$

The lemma is now proved by using (3.9) and (3.10) to bound (3.8) in calculating the $L^{2}$ norm. 


\section{Orders of convergence}

Section 3 can be used to prove the smoothness properties of the solution to the boundary integral equation. The results are known, and may be proved by using regularity results for the solution to Laplace's equation. However the situation can be made more elementary by working directly with the integral equation.

For a vector $\alpha=\left(\alpha_{1}, \ldots, \alpha_{m}\right)$ define the Banach space $\mathcal{C}_{\alpha}^{k}(\Gamma)$ to be the set of vector functions $u=\left(u_{1}, \ldots, u_{m}\right)$ such that for each $i, u_{i} \in \mathcal{C}_{\alpha_{i-1}}^{k}\left(\Gamma_{i}^{-}\right) \cap \mathcal{C}_{\alpha_{i}}^{k}\left(\Gamma_{i}^{+}\right)$. The norm $\|\cdot\|_{k, a}$ is defined as the maximum of the norms of the $u_{i}$ in $\mathcal{C}_{\alpha_{i-1}}^{k}\left(\Gamma_{i}^{-}\right)$ and $\mathcal{C}_{\alpha_{i}}^{i}\left(\Gamma_{i}^{+}\right), i=1, \ldots, m$. The space of functions for which $u_{i} \in \mathcal{C}\left(\bar{\Gamma}_{i}\right)$ is denoted $\mathcal{C}(\Gamma)$, while if $u_{i} \in L^{2}\left(\Gamma_{i}\right)$ the corresponding space is $L^{2}(\Gamma)$.

Let $\bar{\alpha}=\left(\bar{\alpha}_{1}, \ldots, \bar{\alpha}_{m}\right), \quad \bar{\alpha}_{i}=1 /\left(1+\left|\chi_{i}\right|\right)$. It is known (Grisvard [15] or Kondrat'ev [18]) that in a neighbourhood of $s_{i}$, the solution $u$, to (2.3) contains singularities of the form $\left(s-s_{i}\right)^{\bar{\alpha}_{i}}$ even if $g$ is smooth. Thus $u \in \mathcal{C}_{\bar{\alpha}}^{k}(\Gamma)$. We prove directly the weaker result that $u \in \mathcal{C}_{\alpha}^{k}(\Gamma)$ provided $\alpha_{i}<\bar{\alpha}_{i}$ for each $i$.

LEMMA 4. (a) $\mathscr{I}+\mathcal{G}$ has a bounded inverse on $L^{2}(\Gamma)$

(b) $\mathscr{G}+\mathcal{G}$ has a bounded inverse on $\mathcal{C}_{\alpha}^{k}(\Gamma)$ provided $\alpha_{i}<\bar{\alpha}_{i}$ for each $i$.

Proof. The results of section 3 are readily modified to prove that $\|\Re\|<1$ on $L^{2}(\Gamma)$ and hence $\mathscr{g}+\Re$ has a bounded inverse on $L^{2}(\Gamma)$. Similarly, $\mathscr{G}+\Re$ has a bounded inverse on $\mathcal{C}(I)$. A more complicated modification of the results of Section 3 shows $9+\mathcal{R}$ has a bounded inverse on $\mathcal{C}_{\alpha}^{k}(\Gamma)$. (An integral equation is found for a function coinciding with $\left(s_{i}-s\right)^{1-\alpha_{i}} D u_{i}$ and $\left(s-s_{i}\right)^{1-\alpha_{i}} D u_{i+1}$ near $s_{i}$. The details are omitted.)

As $\mathscr{K}(G+\Re)^{-1}$ is compact, the Fredholm alternative shows that (a) or (b) can be proved by showing the homogeneous equation is uniquely solvable. But if $u_{0} \in L^{2}(\Gamma)$ solves $(G+\mathcal{G}) u_{0}=0$, then $(\mathscr{G} \mathscr{R}) u_{0}=-\mathscr{K} u_{0}$ and $\mathcal{K} u_{0} \in \mathcal{C}_{k}^{k}(\Gamma)$ for all $k \geqslant 1$. Whence $u_{0} \in \mathcal{C}_{\alpha}^{k}(\Gamma)$ for all $\alpha$ with $\alpha_{i}<\bar{\alpha}_{i}$. Thus it suffices to show that

$$
(\mathscr{G}+\mathcal{S}) u_{0}=0, \quad u_{0} \in \mathcal{C}_{\alpha}^{k}(\Gamma)
$$

implies $u_{0}=0$.

Let $U_{0}$ be the double layer potential of $u_{0}$. The standard arguments (Kellogg [17] for example) can now be modified to accommodate the corners of $\Gamma$ and used to show $U_{0}=0$. Thus it may be shown (using $u_{0} \in \mathcal{C}_{\alpha}^{2}(\Gamma)$ ) that

(i) $U_{0}$ is harmonic in $\mathbf{R}^{2} \backslash \Gamma$,

(ii) $\left.U_{0}\right|_{\Gamma}$ has a continuous extension, $U_{0}^{(\text {int) }}$, to $\bar{\Omega} \backslash\left\{s_{1}, \ldots, s_{m}\right\}$,

(iii) $U_{0} \mid \bar{\Omega}^{c}$ has a continuous extension, $U_{0}^{\text {(ext) }}$, to $\Omega^{c} \backslash\left\{s_{1}, \ldots, s_{m}\right\}$, 
(iv) $\nabla U_{0}$ is continuous on $\mathbf{R}^{2} \backslash\left\{s_{1}, \ldots, s_{m}\right\}$, and

(v) $U_{0}(x)=O(1 /|x|), \nabla U_{0}(x)=O\left(1 /|x|^{2}\right)$ as $|x| \rightarrow \infty$.

As $u_{0}$ satisfies (4.1), $U_{0}^{\text {(int) }}=0$ on $\Gamma \backslash\left\{s_{1}, \ldots, s_{m}\right\}$ and (i), (ii) and the maximum principle imply $U_{0}^{(\text {int })}=0$. Hence $\left(U_{0}\right)_{\nu}=0$ on $\Gamma \backslash\left\{s_{1}, \ldots, s_{m}\right\}$ and the uniqueness of the exterior Neumann problem (for conditions (v)) implies $U_{0}^{(e x t)}=0$. But as $u_{0}=U_{0}^{(\text {int) }}-U_{0}^{(\text {ext) }}$ on $\Gamma \backslash\left\{s_{1}, \ldots, s_{m}\right\}$ the lemma is proved.

Having established the existence of a unique solution to the boundary integral equation in $L^{2}(\Gamma)$, the existence of a Galerkin approximate solution is immediate. The regularity result in part (b) is necessary to extend Lemma 3 , and prove high order convergence for a graded mesh.

Let $q_{1}, \ldots, q_{m}$ be positive real numbers. For any integer $n$, partition each side $\Gamma_{i}$ of $\Gamma$ by the mesh points $\left\{\sigma_{i j}\right\}$ defined by

$$
\sigma_{i j}= \begin{cases}s_{i-1}+\frac{1}{2}\left(\frac{j}{n}\right)^{q_{i-1}}\left(s_{i}-s_{i-1}\right), & 0 \leqslant j \leqslant n, \\ s_{i}-\frac{1}{2}\left(\frac{2 n-j}{n}\right)^{q_{i}}\left(s_{i}-s_{i-1}\right), & n \leqslant j \leqslant 2 n .\end{cases}
$$

Let $S_{n}$ denote the set of piecewise polynomials of degree $r$ with break points $\left\{\sigma_{i j}\right\}$. In practice the number of mesh points on each side would not be the same, and a more reasonable strategy would be to let this vary in proportion to the length of the side. However this makes no difference to the order of convergence results.

As previously, $P_{n}$ denotes the orthogonal projection of $L^{2}(\Gamma)$ onto $S_{n}$. The Galerkin approximate solution, $u_{n}$, to $(2.3)$ is defined by

$$
\left(9+P_{n} \mathcal{G}\right) u_{n}=P_{n} f
$$

and the iterated Galerkin solution, $u_{n}^{*}$, by

$$
u_{n}^{*}=f-\mathcal{G} u_{n} \text {. }
$$

(See Lin Qun [20], Sloan [23] and the references there.)

THEOREM 5. Suppose for each $i, q_{i}>(r+1) /\left(\bar{\alpha}_{i}+\frac{1}{2}\right)$. Then for $n$ sufficiently large the Galerkin solution, $u_{n}$, is uniquely defined by 4.2 and

$$
\left\|u_{n}-u\right\| \leqslant O\left(\frac{1}{n^{r+1}}\right)
$$

Proof. It is a standard result (Atkinson [2]) that $u_{n}$ is defined for $n$ sufficiently large and

$$
\left\|u_{n}-u\right\| \leqslant C\left\|P_{n}^{\prime} u\right\| .
$$

Thus the theorem follows by the remarks leading to equation (3.7). 
THEOREM 6. Suppose for each $i, q_{i}>(2 r+2) /\left(\bar{\alpha}_{i}+\frac{1}{2}\right)$. Then for $n$ suitably large

$$
\left\|u_{n}^{*}-u\right\| \leqslant O\left(1 / n^{2 r+2}\right) \text {. }
$$

Proof. It is simple to see that $\left(g+\mathcal{G} P_{n}\right) u_{n}^{*}=f$. Hence

$$
\left\|u_{n}^{*}-u\right\| \leqslant C\left\|\mathcal{G} P_{n}^{\prime} u\right\|
$$

provided $\left(\mathscr{G}+\mathcal{G} P_{n}\right)$ is invertible with $\left\|\left(G+\mathcal{G} P_{n}\right)^{-1}\right\|$ bounded uniformly with respect to $n$. But this follows because $\mathscr{K} P_{n}\left(\mathscr{G}+\Re P_{n}\right)^{-1}$ is a sequence of collectively compact operators approximating $\mathscr{K}(G+\mathcal{R})^{-1}$ (Anselone [1]). Now $\left\|\Re P_{n}^{\prime} u\right\| \leqslant O\left(1 / n^{(2 r+2)}\right)$ by suitable application of the proof of Lemma 3 , and $\left\|\mathscr{K} P_{n}^{\prime} u\right\| \leqslant O\left(1 / n^{(2 r+2)}\right)$ is known because $\mathscr{K}$ has a smooth kernel (Chandler [8], Lin Qun [20]).

We can now see the effect of grading the mesh on the order to which the solution to the original Dirichlet problem may be found. Suppose $U(x), x \in \Omega^{0}$ is required. Using $G_{x}(\xi)^{\prime}$ to denote $G(x, \xi)^{\prime}$, the approximation $U_{n}$ defined by

$$
U_{n}(x)=\left(\mathfrak{V} u_{n}\right)(x)=\left(G_{x}^{\prime}, u_{n}\right)=\int_{\Gamma} G_{x}^{\prime}(\xi) u_{n}(\xi) d \xi
$$

can be used. Then

$$
\begin{aligned}
U_{n}(x)-U(x) & =\left(G_{x}^{\prime}, u_{n}-u\right)=\left(G_{x}^{\prime}, u_{n}-P_{n} u\right)+\left(G_{x}^{\prime}, P_{n}^{\prime} u\right) \\
& =\left(P_{n} G_{x}^{\prime}, u_{n}^{*}-u\right)+\left(P_{n}^{\prime} G_{x}^{\prime}, P_{n}^{\prime} u\right)
\end{aligned}
$$

(since $P_{n} u_{n}^{*}=u_{n}$ ). As $x \in \Omega, G_{x}^{\prime}$ is smooth on each $\Gamma_{i}$ and both terms of (4.5) are of order $2 r+2$, provided $q_{i}>(2 r+2) /\left(\bar{\alpha}_{i}+\frac{1}{2}\right)$. As $x$ approaches $\Gamma, G_{x}^{\prime}$ becomes singular and the order of convergence that can be proved for $U_{n}-U$ in the $L^{2}(\bar{\Omega})$ norm is reduced. (To at best order $r+3 / 2$.)

An alternative approximation to $U$ is $U_{n}^{*}$ defined by

$$
U_{n}^{*}(x)=\int_{\Gamma} G_{x}^{\prime} u_{n}^{*}=\left(\mathcal{V} u_{n}^{*}\right)(x) .
$$

Since $\mathfrak{V}$ is a bounded operator from $L^{2}(\Gamma)$ to $L^{2}(\bar{\Omega})$. (This fact may be proved using Lemma 1), we deduce

$$
\left\|U_{n}^{*}-U\right\|_{L^{2}(\bar{\Omega})} \leqslant C\left\|u_{n}^{*}-u\right\| \leqslant O\left(1 / n^{2 r+2}\right)
$$

provided $q_{i}>(2 r+2) /\left(\bar{\alpha}_{i}+\frac{1}{2}\right)$. Thus a piecewise linear approximation to $u$ can be used to recover an order 4 approximation to $U$.

There are two directions for the extension of Theorem 6:

(i) when errors are measured in the uniform norm, and

(ii) when the orthogonal projection $P_{n}$ is replaced by an interpolatory projection and $u_{n}^{*}$ becomes the (more practical) product integration approximation. 
In both (i) and (ii) the order of convergence that should occur may be established by modest generalizations of Lemma 3 . The difficulty is to prove stability results analogous to Theorem 5. For (i) superconvergence techniques are useful, and this is discussed in Chandler [10]. In case (ii) only limited results are available, principally for lower order methods. The best result obtained used the stability theorems in Atkinson and de Hoog [3, 4] to show that a suitable grading restores the order 4 convergence of the two-point Gaussian product integration method.

Finally we make two observations about Theorems 5 and 6 which are relevant in developing software to solve two dimensional potential problems. The first is that the grading of the mesh depends on the degree of the piecewise polynomials to be used. Thus gradings based on conformal mapping arguments which depend only on the angle at the corner (see Jaswon and Symm [16] page 186) may not be entirely satisfactory. Secondly it would be clearly desirable to have an appropriately graded mesh generated automatically. Advantage could then be taken of unexpected smoothness of the solution near a corner, or compensation made for near singular behaviour of $u$ away from a corner. However, an appropriate grading will not be found by looking only at the behaviour of the solution (as is done in Bubuska and Rheinboldt [5] or White [24] for differential equations, and Chandler [9] for a simpler integral equation). For even if $u$ is smooth and $u \in \mathcal{C}_{\alpha}^{r+1}(I), \alpha=r+1$, Lemma 3 shows that the mesh must still be graded so that $q>(2 r+2) /(r+3 / 2)$ before full superconvergence is restored. If only $u_{n}$ is examined (to approximate $D^{r+1} u$ or $u_{n}-u$ for example), a quasi-uniform mesh results, and the advantages of full superconvergence will not be available.

\section{Acknowledgements}

I thank Frank de Hoog for telling me about Fourier transforms, and Ken Atkinson for improving the exposition significantly.

\section{References}

[1] P. M. Anselone, Collectively compact operator approximation theory (Prentice-Hall, Englewood Cliffs, N. J., 1971).

[2] K. E. Atkinson, A survey of numerical methods for the solution of Fredholm integral equations of the second kind (SIAM, 1976).

[3] K. E. Atkinson and F. R. de Hoog, "Collocation methods for a boundary integral equation on a wedge", in Treatment of integral equations by numerical methods (eds. C.T.H. Baker and G. F. Miller), (Academic Press, 1983).

[4] K. E. Atkinson and F. R. de Hoog, "The numerical solution of Laplace's equation on a wedge", IMA J. Numer. Anal., (to appear). 
[5] I. Babuska and W. C. Rheinboldt, "Error estimates for adaptive finite element computations", SIAM J. Numer. Anal. 15 (1978), 736-754.

[6] C. T. H. Baker, The numerical treatment of integral equations (Oxford University Press, 1977).

[7] C. A. Brebbia, Progress in boundary element methods, Vol. 1 (Pentech Press, 1981).

[8] G. A. Chandler, "Superconvergence for second kind integral equations" in The application and numerical solution of integral equations (eds. R. S. Anderssen et al.), (Sijthoff and Noordhoff, 1980), 103-117.

[9] G. A. Chandler, "Superconvergence of numerical solutions to second kind integral equations", Ph.D. Thesis, Australian National University, 1979.

[10] G. A. Chandler, "Superconvergent approximations to the solution of a boundary integral equation on polygonal domains", (in preparation).

[11] S. Christiansen and E. B. Hansen, "Numerical solution of boundary problems through integral equations", Z. Angew. Math. Mech. 58 (1978), T14-T25.

[12] C. W. Cryer, "The solution of the Dirichlet problem for Laplace's equation when the boundary data is discontinuous and the domain has a boundary which is of bounded rotation by means of the Lebesgue-Stieltjes integral equation for the double layer potential", Technical Report 99, Computer Sciences Department, University of Wisconsin (1970).

[13] M. Djaoua, "A method of calculation of lifting flows around two dimensional corner shaped bodies", Math. Comp. 36 (1981), 405-521.

[14] I. G. Graham, "Galerkin methods for second kind integral equations with singularities", Math. Comp. 39 (1982), 519-533.

[15] P. Grisvard, "Behaviour of the solutions of an elliptic boundary value problem in a polygonal or polyhedral domain", in Numerical solution of partial differential equations-III, Synspade, 1975 (ed. B. Hubbard), (Academic Press, 1976), 207-247.

[16] M. A. Jaswon and G. I. Symm, Integral equation methods in potential theory and electrostatics (Academic Press, 1977).

[17] O. D. Kellogg, Foundations of potential theory (J. Springer, 1929; Dover, 1954).

[18] V. A. Kondrat'ev, "Boundary problems for elliptic equations in domains with conical or angular points", Trans. Moscow Math. Soc. 16 (1967), 227-313.

[19] J. Král, "Integral operators in potential theory", in Lecture Notes in Mathematics 823, (Springer-Verlag, 1980).

[20] Lin Qun, "Some problems about the approximate solution for operator equations", Acta Math. Sinica 22 (1979), 219-230.

[21] J. R. Rice, "On the degree of convergence of nonlinear spline approximation" in Approximation with special emphasis on spline functions (ed. I. J. Schoenberg), (Academic Press, New York 1969).

[22] C. Schneider, "Product integration for weakly singular integral equations", Z. Angew. Math. Mech. 61 (1981), T317-319.

[23] I. H. Sloan, "A review of numerical methods for Fredholm equations of the second kind" in The application and numerical solution of integral equations (eds. R. S. Anderssen et al.), (Sijthoff and Noordhoff, 1980), 51-74.

[24] A. B. White, "On selection of equidistributing meshes for two point boundary value problems, SIAM. J. Numer. Anal. 16 (1979), 472-502. 